\title{
Wireless Network Design via 3-Decompositions
}

\author{
Zeev Nutov \\ The Open University of Israel \\ nutov@openu.ac.il
}

\author{
Ariel Yaroshevitch* \\ The Open University of Israel \\ ariel@walesltd.co.il
}

Key-words: Wireless Networks, Steiner Trees, Approximation Algorithms.

\begin{abstract}
We consider some network design problems with applications for wireless networks. The input for these problems is a metric space $(X, d)$ and a finite subset $U \subseteq X$ of terminals. In the Steiner Tree with Minimum Number of Steiner Points (STMSP) problem, the goal is to find a minimum size set $S \subseteq X-U$ of points so that the unit-disc graph of $S+U$ is connected. Let $\Delta$ be the smallest integer so that for any finite $V \subseteq X$ for which the unit-disc graph is connected, this graph contains a spanning tree with maximum degree $\leq \Delta$. The best known approximation ratio for STMSP was $\Delta-1[10]$. We improve this ratio to $\lfloor(\Delta+1) / 2\rfloor+1+\varepsilon$.

In the Minimum Power Spanning Tree (MPST) problem, $V=X$ is finite, and the goal is to find a "range assignment" $\{p(v): v \in V\}$ on the nodes so that the edge set $\{u v \in E: d(u v) \leq \min \{p(u), p(v)\}\}$ contains a spanning tree, and $\sum_{v \in V} p(v)$ is minimized. We consider a particular case $\{0,1\}$-MPST of MPST when the distances are in $\{0,1\}$; here the goal is to find a minimum size set $S \subseteq V$ of "active" nodes so that the graph $\left(V, E_{0}+E_{1}(S)\right)$ is connected, where $E_{0}=\{u v: d(u v)=0\}$, and $E_{1}(S)$ is the set the edges in $E_{1}=\{u v: d(u v)=1\}$ with both endpoints in $S$. We will show that the $(5 / 3+\varepsilon)$-approximation scheme for MPST of [1] achieves a ratio $3 / 2$ for $\{0,1\}$-distances. This answers an open question posed in [9].
\end{abstract}

*Part of this work was done as a part of author's M.Sc. Thesis at The Open University of Israel. 


\section{Introduction}

\subsection{Problems considered}

A large research effort is focused on developing algorithms for finding a "cheap" network that satisfies some prescribed requirements. In wired networks, where connecting any two nodes incurs a cost, many problems can be cast as finding a subgraph of minimum cost. Fundamental problems in this setting are Minimum Spanning Tree and Minimum Steiner Tree. In wireless networks, every transmitter usually has some fixed range, and the location of "active" transmitters determines the resulting communication network. We consider "activating" minimum number of transmitters, so that the resulting communication network is connected. If the range of the transmitters is fixed, our goal is to add minimum number of transmitters. If some of the transmitters are already present ("activated"), or if some of the terminals are already connected by a wired infrastructure, our goal is to "activate" minimum number of (additional) transmitters. The following two types of problems arise in the setting when we wish to connect all or some terminals of the network.

Definition 1.1 Let $(X, d)$ be a metric space and let $V \subseteq X$. The unit-disk graph of $V$ has node set $V$ and edge set $\{u v: u, v \in V, d(u, v) \leq 1\}$.

Steiner Tree with Minimum Number of Steiner Points (STMSP)

Instance: A metric space $(X, d)$ and a finite set $U \subseteq X$ of terminals.

Objective: Find a min-size set $S \subseteq X-U$ so that the unit-disk graph of $U+S$ is connected.

Definition 1.2 Let $G=(V, E)$ be a graph with edge distances $\{d(e): e \in E\}$. For $v \in V$, the power $p(v)=p_{d}(v)$ of $v$ in $G$ (w.r.t. $d$ ) is the maximum length of an edge incident to $v$ in $G$ (or zero, if no such edge exists). The power $p(G)=\sum_{v \in V} p(v)$ of $G$ is the sum of the powers of its nodes.

In the Minimum Power Spanning Tree (MPST) problem, we are given a graph $G=(V, E)$ with edge distances $\{d(e): e \in E\}$, and the goal is to find a minimum power spanning tree of $G$. We consider a particular case of MPST when all the distances are in $\{0,1\}$. In the applications described, this is essentially the case when the power of the transmitters is fixed. This case can be cast as follows:

$\{0,1\}$-MPST

Instance: A graph $G=\left(V, E_{0}+E_{1}\right)$.

Objective: Find a minimum size set $S \subseteq V$ of "active" nodes so that the graph $\left(V, E_{0}+E_{1}(S)\right)$ is connected, where $E_{1}(S)$ is the set the edges in $E_{1}$ with both endpoints in $S$. 


\subsection{Previous and related work}

Both STMSP and $\{0,1\}$-MPST are known to be NP-hard, and arise in various wireless network design problems, c.f. $[1,9,3,10]$. STMSP was studied both in the Euclidean plane and in general metric spaces. In the latter case, the ratio is usually expressed in terms of the following parameter. Let $\Delta$ be the smallest integer so that for any finite $V \subseteq X$ for which the unit-disc graph is connected, this graph contains a spanning tree with maximum degree $\leq \Delta$. Măndoiu and Zelikovsky [10] gave a $(\Delta-1)$-approximation algorithm for STMSP for any metric space. Robins and Salowe [12] proved that for metric spaces defined on $X=R^{k}$ with the distances $d_{p}(x, y)=\left(\sum_{i=1}^{k}\left|x_{i}-x_{j}\right|^{p}\right)^{1 / p}$ the parameter $\Delta$ equals the maximum number of "independent" points that can be placed on the surface of a unit sphere, such that the distance between each pair of points is strictly more than one. For Euclidean distances we have $\Delta=5$ in $R^{2}$ and $\Delta=11$ in $R^{3}$, but the exact values of $\Delta$ are not known for all $p, k$. The currently best approximation ratio to the STMSP in $R^{2}$ is $2.5+\varepsilon$ by [3].

In finite metric spaces, STMST is equivalent to the variant of the Node Weighted Steiner Tree problem when all terminals have costs 0 and the other nodes have cost 1 . Klein and Ravi [6] proved that this variant is Set-Cover hard to approximate, and gave an $O(\ln n)$ approximation algorithm for general weights. Hence up to constants, even for finite metric spaces, the ratio $O(\ln n)$ of Klein and Ravi [6] is the best possible guarantee unless $\mathrm{P}=\mathrm{NP}$. Note however, that this does not exclude constant ratios for metric spaces with small $\Delta$.

General min-power connectivity problems were studied extensively, see for example [4, $1,2,5,7]$ for only a small sample of papers in this area. The best known ratio for MPST is $5 / 3+\varepsilon[1]$. The particular case $\{0,1\}$-MPST was first considered in [8], where it was called Max-power Users. A fast 5/3-approximation algorithm for $\{0,1\}$-MPST was developed in [9]. In [9] it was also shown that the ratio $5 / 3$ of their algorithm is tight, and was posed an open problem to get a ratio better than $5 / 3$.

\subsection{Our results}

The previously best ratio for STMSP in metric spaces with finite $\Delta$ was $\Delta-1$ [10]. Our main result improves this ratio for $\Delta \geq 6$, assuming that STMSP instances with at most 3 terminals can be solved in polynomial time.

Theorem 1.1 STMSP admits a $(\lfloor(\Delta+1) / 2\rfloor+1+\varepsilon)$-approximation scheme.

For $\{0,1\}$-MPST, we resolve the open problem from [9] of achieving a ratio better than $5 / 3$, by proving that the $(5 / 3+\varepsilon)$-approximation scheme for MPST of [1] achieves the ratio 
$3 / 2+\varepsilon$ for 0,1 distances.

Theorem 1.2 \{0,1\}-MPST admits a 3/2-approximation algorithm.

\section{Approximating via connected 3-decompositions}

We use the decomposition method developed by Zelikovsky [13] and Prömel and Steger [11] for the Minimum-Cost Steiner Tree problem. This method reduces "Steiner-Tree" problems, with some loss in the approximation ratio, to a Min-Costs Connected Spanning Sybhypergraph problem in 3-hypergraphs. The later problem admits a polynomial time algorithm for bounded costs and a FPTAS for general costs [11]. In our case, to prove Theorem 1.1, we use a similar reduction, and show that the loss in the approximation ratio is $\lfloor(\Delta+1) / 2\rfloor+1$ for STMSP, and $3 / 2$ for $\{0,1\}$-MPST (the second bound is tight). More precisely, given an instance $\mathcal{I}$ of STMSP or of $\{0,1\}$-MPST, we construct in polynomial time an instance $\mathcal{E}, c$ of Min-Costs Connected Spanning Subhypergraph in the 3-hypergraph $\mathcal{E}$, such that $\operatorname{opt}(\mathcal{E}, c) \leq \rho \cdot \operatorname{opt}(\mathcal{I})$, where $\rho=\lfloor(\Delta+1) / 2\rfloor+1$ for STMSP and $\rho=3 / 2$ for $\{0,1\}$ MPST, and such that for any feasible solution $\mathcal{E}^{\prime}$ to $\mathcal{E}, c$ corresponds a feasible solution $T$ to $\mathcal{I}$ with $p(T) \leq c\left(\mathcal{E}^{\prime}\right)$. Formally, this method applies for the following class of problems:

Generalized Steiner Tree

Instance: A (possibly infinite) graph $G=(V, E)$, a finite set $U \subseteq V$ of terminals, and a monotone non-decreasing subadditive cost function $c$ on finite subgraphs of $G$.

Objective: Find a minimum-cost connected (finite) subtree $T$ of $G$ containing $U$.

The graph $G$ and the cost function $c$ may not be given explicitly, but we require that some queries related to $G, c$ can be answered in polynomial time. Specifically, we will assume that instances with at most 3 terminals can be solved in polynomial time.

Definition 2.1 A $k$-decomposition of a set $U$ is a collection $\mathcal{E}$ of subsets of $U$, of size at most $k$ each, so that their union is $U$. A decomposition $\mathcal{E}$ is connected if the hypergraph $(U, \mathcal{E})$ is connected. Given a cost function c on subsets of $U$, the cost of a $k$-decomposition $\mathcal{E}$ is $c(\mathcal{E})=\sum_{U^{\prime} \in \mathcal{E}} c\left(U^{\prime}\right)$.

Definition 2.2 Given a tree $T$ with a set $U$ of terminals, and a cost function on the subtrees of $T$, the $T$-cost $c_{T}\left(U^{\prime}\right)$ of $U^{\prime} \subseteq U$ is the cost of a minimal inclusion subtree $T^{\prime}$ of $T$ containing $U^{\prime}$. The $T$-cost of a $k$-decomposition $\mathcal{E}$ of $U$ is $c_{T}(\mathcal{E})=\sum_{U^{\prime} \in \mathcal{E}} c_{T}\left(U^{\prime}\right)$. Given an instance of Generalized Steiner Tree and a (finite) tree $T$ containing $U$, let $\mathcal{E}_{T}$ be a minimum $T$-cost 3-decomposition of $U$. The 3-decomposition ratio is defined by $\rho=\sup _{T} c\left(\mathcal{E}_{T}\right) / c(T)$ where the supremum is taken over all finite trees $T$ containing $U$. 
The following statement was implicitly proved by Prömel and Steger [11]; for completeness of exposition we provide a short proof.

Theorem 2.1 Generalized Steiner Tree admits a $(\rho+\varepsilon)$-approximation scheme, assuming that for any $U^{\prime} \subseteq U$ with $\left|U^{\prime}\right| \leq 3$ an optimal tree $T\left(U^{\prime}\right)$ containing $U^{\prime}$ can be computed in polynomial time, where $\rho$ is the 3-decomposition ratio.

Proof: The algorithm is as follows.

1. Let $\mathcal{E}=\left\{U^{\prime} \subseteq U:|U| \in\{2,3\}\right\}$.

For every $U^{\prime} \in \mathcal{E}$ compute an optimal subtree $T\left(U^{\prime}\right)$ containing $U^{\prime}$.

2. In the hypergraph $(U, \mathcal{E})$ with edge costs $c\left(U^{\prime}\right)=c\left(T\left(U^{\prime}\right)\right)$, compute a $\left(1+\varepsilon^{\prime}\right)$ approximate solution $\mathcal{E}^{\prime}$ to the Min-Costs Connected Spanning Subhypergraph problem.

3. Return a spanning tree $T$ of the graph $H=\cup\left\{T\left(U^{\prime}\right): U^{\prime} \in \mathcal{E}^{\prime}\right\}$.

Step 1 in the algorithm can be implemented in polynomial time by the assumption. Step 2 is implemented using the algorithm of Prömel and Steger [11]. The computed solution is feasible since the union of the sets in $\mathcal{E}^{\prime}$ is $U$ (so $T$ contains $U$ ), and since $\mathcal{E}^{\prime}$ is connected (so $T$ is connected). We prove the approximation ratio. Let $T^{*}$ be an optimal solution so $c\left(T^{*}\right)=$ opt. Let $\mathcal{E}^{*}$ be a connected 3 -decomposition of $T^{*}$-cost at most $\rho \cdot$ opt; such $\mathcal{E}^{*}$ exists, by the definition of $\rho$. Let $\varepsilon^{\prime}=\varepsilon / \rho$. Then for the tree $T$ computed we have:

$$
c(T) \leq c(H) \leq \sum_{U^{\prime} \in \mathcal{E}^{\prime}} c\left(T\left(U^{\prime}\right)\right) \leq\left(1+\varepsilon^{\prime}\right) \sum_{U^{\prime} \in \mathcal{E}^{*}} c_{T^{*}}\left(U^{\prime}\right) \leq\left(1+\varepsilon^{\prime}\right) \rho \cdot \text { opt }=(\rho+\varepsilon) \cdot \text { opt } .
$$

The first inequality holds since $c$ is non-decreasing, the second holds since $c$ is subadditive. The third inequality holds since $\mathcal{E}^{*}$ is a connected 3 -decomposition while $\mathcal{E}^{\prime}$ is a $\left(1+\varepsilon^{\prime}\right)$ approximate optimal connected 3-decomposition.

Now let us show how our problems fit this setting. In the case of STMSP, the graph $G$ is the unit-disc graph of all points in the metric space $(X, d)$, and here $G$ may be infinite. The cost $c(H)$ of a finite subgraph $H$ of $G$ is the number of non-terminal nodes in $H$. In the case of $\{0,1\}$-MPST, every node is a terminal, and the cost function $c$ is the power $p(H)$ of $H$ (namely, in the case of 0,1 -distances, the number of "activated" nodes in $H$ ). It is known and easy to verify that in both cases $c$ is monotone non-decreasing and subadditive. We will prove that the 3 -decomposition ratio is bounded by $\lfloor(\Delta+1) / 2\rfloor+1$ for STMSP and by $3 / 2$ for $\{0,1\}$-MPST. Furthermore, in the case of $\{0,1\}$-MPST, we will show that it is sufficient to consider only sets in $\mathcal{E}$ so that $c\left(T\left(U^{\prime}\right)\right) \leq 3$. In this case the corresponding Min-Cost 
Connected Spanning Sybhypergraph problem at Step 2 can be solved exactly in polynomial time, and thus the approximation ratio can be reduced from $3 / 2+\varepsilon$ to $3 / 2$.

It remains to justify the assumption that problem instances with at most 3 terminals can be solved in polynomial time. This assumption is clearly valid for $\{0,1\}$-MPST, and in fact for MPST, see [1]. For STMSP, we cannot exclude the possibility that the validity of this assumption depends on the metric space $(X, d)$, but we are not aware of any specific metric space for which this assumption is not valid. It is valid for Euclidean distances in $R^{2}$ (see e.g. [3]), and this easily implies validity also for metric spaces $\left(R^{k}, d_{p}\right)$.

\subsection{3-decomposition ratio for STMSP (Proof of Theorem 1.1)}

Let $T=(V, E)$ be a tree, let $U \subseteq V$ be a set of terminals, and let $S=V-U$. Recall that here the $T$-cost of a subset $U^{\prime} \subseteq U$ of terminals is the number of non-terminals in the inclusion minimal subtree $T^{\prime}$ of $T$ containing $U^{\prime}$. We need to show that there exists a connected 3-decomposition of $U$ so that $c_{T}(\mathcal{E}) /|S| \leq\lfloor(\Delta+1) / 2\rfloor+1$, where $\Delta$ is the maximum degree of the tree. The key observation is:

Lemma 2.2 Let $T=(V, E)$ be a tree, let $U \subseteq V$ be a set of terminals, and let $S=V-U$. Denote $S_{\text {odd }}=\left\{s \in S: \operatorname{deg}_{T}(s)\right.$ is odd $\}$ and suppose that $\min \left\{\operatorname{deg}_{T}(s): s \in S_{\text {odd }}\right\} \geq 2 i+1$ for $i \geq 1$ if $S_{\text {odd }} \neq \emptyset$. Let $\gamma=i /(2 i-1)$ if $S_{\text {odd }} \neq \emptyset$ and $\gamma=1 / 2$ otherwise. Then there exists a connected 3 -decomposition $\mathcal{E}$ of $U$ so that:

$$
c_{T}(\mathcal{E}) \leq \frac{1}{2} \sum_{s \in S} \operatorname{deg}_{T}(s)+\gamma|S|+\frac{1}{2}\left|S_{\text {odd }}\right|
$$

Proof: We may assume that $U=L$ is the set of leaves of $T$. The proof is by induction on $|L|$. The induction base is $|L|=2$. Then $T$ is a path $P$, and let $p$ be its length. The trivial decomposition $\{L\}$ has $T$-cost $p-1$. The first term in the right hand side of (1) equals $p-1$. Hence the statement holds in this case.

Assume that $|L| \geq 3$. Root the tree at an arbitrary node in $U$. Then $T$ has a node $a \in S$ of degree $\geq 3$, so that the subtree $T_{a}$ of $T$ that consists of $a$ and all its descendants is a spider; namely, in $T_{a}$ the degree of every proper descendant of $a$ is at most 2 , while the degree of $a$ is at least 2 . Let $t=\operatorname{deg}_{T}(a)-1 \geq 2$ be the number of leaves in $T_{a}$. Let $u \in L \cap T_{a}$ be the closest leaf to $a$ in $T_{a}$, let $P$ be the au-path in $T_{a}$, and let $p=|P \cap S|-1$. Note that $p \geq 0$, as $a \in P \cap S$. Let $k=\left|S \cap\left(T_{a}-P\right)\right|$. Note that:

$$
k \geq p \cdot(t-1)
$$


Partition the leaves in $T_{a}$ distinct from $u$ into sets of size 2 and possibly one singleton. Obtain a 3-decomposition $\mathcal{E}_{a}$ of $L \cap T_{a}$ by adding $u$ to each part. The $T$-cost of this decomposition is $c_{T}\left(\mathcal{E}_{a}\right)=(p+1) \cdot\lceil(t-1) / 2\rceil+k$, namely:

$$
c_{T}\left(\mathcal{E}_{a}\right)= \begin{cases}(p+1) t / 2+k & \text { if } t \text { is even } \\ (p+1)(t-1) / 2+k & \text { if } t \text { is odd }\end{cases}
$$

Now remove $T_{a}-P$ from $T$; note that no nodes of odd degree are created, and that $a$ becomes a node of degree 2 . It is sufficient to show that the decrease in the right hand side of (1) is at least the $T$-cost $c_{T}\left(\mathcal{E}_{a}\right)$ of the decomposition $\mathcal{E}_{a}$.

- The decrease in $\sum_{s \in S} \operatorname{deg}_{T}(s)$ is $2 k+t-1$, and the decrease in $|S|$ is $k$.

- The decrease in $\left|S_{\text {odd }}\right|$ is 1 if $t$ is even (as then $\operatorname{deg}_{T}(a)$ is odd) and is 0 if $t$ is odd.

Thus the total decrease in the right hand side of (1) is:

$$
\begin{array}{ll}
\frac{1}{2}(2 k+t-1)+\gamma k+1 / 2=k \cdot(1+\gamma)+t / 2 & \text { if } t \text { is even } \\
\frac{1}{2}(2 k+t-1)+\gamma k=k \cdot(1+\gamma)+(t-1) / 2 & \text { if } t \text { is odd } .
\end{array}
$$

Thus it is sufficient to show that:

$$
\begin{array}{ll}
k(1+\gamma)+t / 2 \geq(p+1) t / 2+k & \text { if } t \text { is even } \\
k(1+\gamma)+(t-1) / 2 \geq(p+1)(t-1) / 2+k & \text { if } t \text { is odd }
\end{array}
$$

And by rearranging terms:

$$
\begin{array}{ll}
\gamma k \geq p t / 2 & \text { if } t \text { is even } \\
\gamma k \geq p(t-1) / 2 & \text { if } t \text { is odd . }
\end{array}
$$

Consequently, as $k \geq p(t-1)$ and $p \geq 0$ it is sufficient to require:

$$
\begin{array}{ll}
\gamma(t-1) \geq t / 2 & \text { if } t \text { is even } \\
\gamma(t-1) \geq(t-1) / 2 & \text { if } t \text { is odd }
\end{array}
$$

The second inequality holds as $\gamma=i /(2 i-1)>1 / 2$. The first inequality is easily verified after observing that if $t$ is even then $\operatorname{deg}_{T}(a)$ is odd, and thus $t=\operatorname{deg}_{T}(a)-1 \geq 2 i$.

Corollary 2.3 $c_{T}(\mathcal{E}) /|S| \leq\lfloor(\Delta+1) / 2\rfloor+1$ holds for the decomposition $\mathcal{E}$ in Lemma 2.2, where $\Delta=\max _{S \in S} \operatorname{deg}_{T}(s)$. Thus the 3 -decomposition ratio for STMST is bounded by $\lfloor(\Delta+$ $1) / 2\rfloor+1$. 

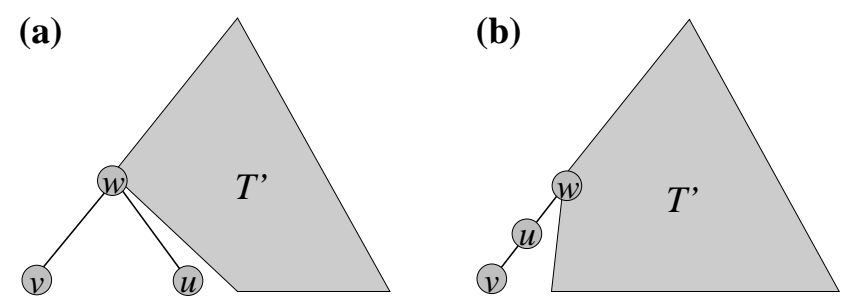

Figure 1: Illustration to the proof of Lemma 2.4 .

Proof: Clearly, $\sum_{s \in S} \operatorname{deg}_{T}(s) \leq|S| \Delta,\left|S_{\text {odd }}\right| \leq|S|$, and $\gamma \leq 1$. Thus from (1) we obtain $c_{T}(\mathcal{E}) \leq|S|(\Delta+3) / 2$. Hence if $\Delta$ is odd then $c_{T}(\mathcal{E}) /|S| \leq\lfloor(\Delta+1) / 2\rfloor+1$. Now suppose that $\Delta$ is even; we need to prove that then $c_{T}(\mathcal{E}) \leq|S|(\Delta / 2+1)$. Let $q$ be the fraction of nodes in $S$ whose degree in $T$ is at most $\Delta-1$. Namely, $\left|\left\{s \in S: \operatorname{deg}_{T}(s) \leq \Delta-1\right\}\right|=q \cdot|S|$. Note that $\left|S_{\text {odd }}\right| \leq q \cdot|S|$. By (1) we get:

$c_{T}(\mathcal{E}) \leq \frac{1}{2} \sum_{s \in S} \operatorname{deg}_{T}(s)+|S|+\frac{1}{2}\left|S_{\text {odd }}\right| \leq \frac{|S|}{2}[\Delta(1-q)+(\Delta-1) q]+|S|+\frac{q|S|}{2}=\frac{|S|}{2}(\Delta+2)$.

The proof of Theorem 1.1 is complete.

\subsection{3 -decomposition ratio for $\{0,1\}$-MPST (Proof of Theorem 1.2)}

Recall that here every node is a terminal (so $V=U$ ), that the distances are in 0,1 , and the $T$-cost of a subset $U^{\prime} \subseteq U$ is the the power (namely, the number of nodes incident to edges of $E_{1}$ ) in the inclusion minimal subtree $T^{\prime}$ of $T$ containing $U^{\prime}$.

Lemma 2.4 For 0,1 distances, any tree $T$ with with at least 4 nodes admits a connected 3-decomposition $\mathcal{E}$ such that $p_{T}(\mathcal{E})=3 / 2 \cdot p(T)-1$; moreover, $p\left(T\left(U^{\prime}\right)\right) \leq 3$ for all $U^{\prime} \in \mathcal{E}$. Thus the 3-decomposition ratio for MPST is bounded by $3 / 2$.

Proof: Note that taking into the decomposition every edge of cost 0 does not increase the power of the decomposition. Hence it is sufficient to prove the statement for every connected component of $T-\{e \in T: d(e)=0\}$, namely, for the case of unit distances. We prove the statement by induction on the number $n$ of nodes in $T$. The induction base is $n \in\{4,5\}$, in which case the statement is easily verified.

Assume that the statement holds for any tree with at most $n-1$ nodes, and we will prove it for a tree $T$ with $n$ nodes, $n \geq 6$. It is easy to see that $T$ has a triplet $u, v, w$ of nodes so that $v$ is a leaf and either: $u$ is a leaf and $v w, w u \in T$ (Fig. 1(a)), or $\operatorname{deg}_{T}(u)=2$ and $v u, u w \in T$ (Fig. 1(b)). 
Let $T^{\prime}=T-\{u, v\}$. By the induction hypothesis there exists a connected 3-decomposition $\mathcal{E}^{\prime}$ of $T^{\prime}$ as in the lemma; namely, $p\left(\mathcal{E}^{\prime}\right)=3 / 2 \cdot p\left(T^{\prime}\right)-1$ and the $T^{\prime}$-cost of every $U^{\prime} \in \mathcal{E}^{\prime}$ is at most 3 . Let $\mathcal{E}$ be obtained by adding to $\mathcal{E}^{\prime}$ the subtree of $T$ induced by $u, v, w$. Clearly, $\mathcal{E}$ is a connected 3-decomposition of $T$, and $p(T)=p\left(T^{\prime}\right)+2$ and the power of the subtree induced by $u, v, w$ is 3 . Consequently,

$$
p(\mathcal{E})=p\left(\mathcal{E}^{\prime}\right)+3=3 / 2 \cdot p\left(T^{\prime}\right)-1+3=3 / 2 \cdot\left(p\left(T^{\prime}\right)+2\right)-1=3 / 2 \cdot p(T)-1 .
$$

The proof of Theorem 1.2 is complete.

\section{References}

[1] E. Althaus, G. Calinescu, I. Măndoiu, S. Prasad, N. Tchervenski, and A. Zelikovsky. Power efficient range assignment for symmetric connectivity in static ad hoc wireless networks. Wireless Networks, 12(3):287-299, 2006.

[2] G. Calinescu, S. Kapoor, A. Olshevsky, and A. Zelikovsky. Network lifetime and power assignment in ad hoc wireless networks. In ESA, pages 114-126, 2003.

[3] D. Chen, D.-Z. Du, X.-D. Hu, G.-H. Lin, L. Wang, and G. Xue. Approximations for Steiner trees with minimum number of steiner points. Theoretical Comput. Science, 262(1):83-99, 2001.

[4] A. Clementi, P. Penna, and R. Silvestri. Hardness results for the power range assignmet problem in packet radio networks. In APPROX, pages 197-208, 1999.

[5] M. T. Hajiaghayi, G. Kortsarz, V. S. Mirrokni, and Z. Nutov. Power optimization for connectivity problems. Math. Program., 110(1):195-208, 2007.

[6] P. Klein and R. Ravi. A nearly best-possible approximation algorithm for node-weighted steiner trees. J. Algorithms, 19(1):104-115, 1995.

[7] G. Kortsarz, V. Mirrokni, Z. Nutov, and E. Tsanko. Approximating minimum-power degree and connectivity problems. In LATIN, pages 423-435, 2008. To appear in Algorithmica.

[8] E. Lloyd, R. Liu, M. Marathe, R. Ramanathan, and S. Ravi. Algorithmic aspects of topology control problems for ad hoc networks. MONET, 10(1-2):19-34, 2005. 
[9] E. Lloyd, R. Liu, and S. Ravi. Approximating the minimum number of maximum power users in ad hoc networks. Mobile Networks and Applications, 11:129-142, 2006.

[10] I. I. Măndoiu and A. Z. Zelikovsky. A note on the MST heuristic for bounded edgelength Steiner trees with minimum number of Steiner points. Information Procassing Letters, 75(4):165-167, 2000.

[11] H. J. Prömel and A. Steger. A new approximation algorithm for the Steiner tree problem with performance ratio 5/3. J. of Algorithms, 36(1):89-101, 2000.

[12] G. Robins and J. S. Salowe. Low-degree minimum spanning trees. Discrete Comput. Geom., 14:151-165, 1995.

[13] A. Zelikovsky. An 11/6-approximation algorithm for the network Steiner problem. Algorithmica, 9(5):463-470, 1993. 